\title{
Ein neuer Impuls für eine europäische Energiepolitik? Ergebnisse des EU-Frühjahrsgipfels 2007
}

\author{
Raphael Sauter und Katherina Grashof*
}

Das auch als ,Energiegipfel ‘ bezeichnete Frühjahrstreffen der europäischen Staats- und Regierungschefs im März 2007 wurde von manchen als ein wichtiger - wenn nicht sogar der entscheidende - Schritt in Richtung einer gemeinsamen europäischen Energiepolitik gewertet. Aus verschiedenen Gründen wird ein gemeinschaftliches Vorgehen in diesem Bereich für notwendig erachtet. Die unzureichende Umsetzung des europäischen Energiebinnenmarktes führt zu überhöhten Energiepreisen und schränkt die Wettbewerbsfähigkeit der europäischen Volkswirtschaft ein. Gleichzeitig müssen im europäischen Energiesektor bereits heute Rahmenbedingungen geschaffen werden, die notwendige Investitionen in Höhe von tausend Milliarden Euro in den nächsten zwanzig Jahren ermöglichen. ${ }^{1}$ Ferner wurden angesichts der Konflikte um Energielieferungen von Russland nach Ostmitteleuropa und Nachfolgestaaten der UdSSR das Problem der Importabhängigkeit und die möglichen Auswirkungen auf die Energieversorgungssicherheit der Europäischen Union vor dem Energiegipfel in den Vordergrund gestellt. Mit der seit Jahresbeginn deutlich intensivierten Debatte um die Eindämmung des Klimawandels rückt schließlich das Thema Treibhausgasemissionen des Energiesektors - sie betragen vier Fünftel der europäischen Emissionen - hoch auf die Agenda der gemeinschaftlich zu verfolgenden Aufgaben. ${ }^{2}$ Wenn die traditionell als Vorreiterin in den internationalen Klimaschutzverhandlungen agierende Europäische Union nicht an Glaubwürdigkeit einbüßen soll, müssen die bestehenden Ziele im Rahmen des Kyoto-Protokolls eingehalten werden und darüber hinaus für die Zukunft ambitionierte Schritte erkennbar sein.

Auch vor diesem Hintergrund hat die britische Ratspräsidentschaft 2005 die Energie- und Klimapolitik ins Zentrum des Europäischen Rates in Hampton Court gestellt, der die Kommission um die Ausarbeitung entsprechender Vorschläge gebeten hat, die Anfang $2007 \mathrm{im}$ sogenannten ,Energiepaket“ vorgelegt wurden und auf dem Gipfeltreffen im März 2007 zur Verabschiedung eines energiepolitischen Aktionsplans geführt haben. Kann dieser Aktionsplan nun als Meilenstein auf dem Weg zu einer europäischen Energiepolitik gewertet werden - ein Politikbereich, in dem die Europäische Kommission seit Jahrzehnten, wenn auch bisher eher erfolglos, eigene Kompetenzen anstrebt? Oder handelt es sich eher um neue Impulse in alten Politikfeldern? Zur Beantwortung dieser Fragen werden die Themen des verabschiedeten Aktionsplans mit Blick auf die jeweils bestehende europäische Rechtslage und ihre bisherige Umsetzung, ihren aktuellen Kontext und bestehende Interessenlagen analysiert und schließlich zentrale Punkte der nun anstehenden Umsetzung herausgearbeitet. Aufgrund der angestrebten integrierten europäischen Energie- und Klimaschutzpolitik wird neben dem Aktionsplan auch der in den Schlussfolgerungen des Rates enthaltene Beschluss zur europäischen Klimapolitik berücksichtigt. In diesem Zusammenhang werden mit Kernenergie,

* Raphael Sauter, wissenschaftlicher Mitarbeiter und Doktorand, University of Sussex, Brighton, UK. Katherina Grashof, Öko-Institut e. V., jetzt Referentin am Bundesamt für Strahlenschutz. Die Autoren danken Herrn Prof. Dr. Uwe Leprich für Kommentare zu einem früheren Entwurf dieses Artikels.

1 Vgl. Europäische Kommission: Grünbuch. Eine europäische Strategie für nachhaltige, wettbewerbsfähige und sichere Energie, $\operatorname{KOM}(2006) 105$ endgültig, S. 3.

2 Europäische Kommission: Mitteilung an den Rat und das Europäische Parlament, Eine Energiepolitik für Europa, $\operatorname{KOM}(2007) 1$ endgültig, S. 3. 
Emissionshandel und emissionsarmer Kohlenutzung weitere zentrale Themen dieser Politikbereiche diskutiert, die zwar in den Schlussfolgerungen des Europäischen Rates wenig Raum einnehmen, für eine umfassende Bewertung der Gipfelbeschlüsse jedoch einzubeziehen sind. Die Gipfelbeschlüsse werden nach einer kurzen Einführung und Vorstellung in folgender Reihenfolge diskutiert: Energiebinnenmarkt, Diversifizierung der Rohstoffversorgung, Energieeffizienz, erneuerbare Energien, Kernenergie sowie klimafreundliche Kohle.

\section{Energiepolitik auf europäischer Ebene}

Aufgrund der fehlenden energiepolitischen Kompetenz bedient sich die Kommission im Bereich der Energiepolitik zwangsläufig ,,vertragliche[r] ,Hilfskonstruktionen ““.3 Dabei bezieht sie sich vor allem auf ihre Kompetenzen in den Bereichen Wettbewerbs-, Binnenmarkt- und Umweltpolitik. Dies erklärt auch eine teilweise fluktuierende Prioritätensetzung im Bereich der Energiepolitik. Der politische Prozess in diesem Politikfeld wurde daher treffend als, von der Seite' durch verschiedene Generaldirektionen (Beispiel Energiebinnenmarktrichtlinie) und ,von oben' durch Initiativen des Europäischen Rates (Beispiel Energiecharta) beschrieben. ${ }^{4}$

Entsprechend variieren auch die zugrunde liegenden Zielsetzungen. In den 1980er und 1990er Jahren war die europäische Energiepolitik von zwei weitgehend separaten Agenden bestimmt, der Wettbewerbs- und der Versorgungssicherheitspolitik. Im Zuge sinkender Ölpreise rückte letztere zeitweise in den Hintergrund und gewann erst angesichts ausbleibender Investitionen infolge von Privatisierung und Liberalisierung in den 1990er Jahren sowie der jüngsten Konflikte an Relevanz. Mit dem allmählichen Bedeutungszuwachs des Ziels Umweltschutz seit den 1980er Jahren traten in das - traditionell von starken und gut mit, ihren' nationalen Regierungen vernetzten Energieunternehmen dominierte - politikprägende Akteursgefüge Verbraucher- und Umweltorganisationen neu hinzu. ${ }^{5}$ Aktuell bezieht sich dieses Ziel vor allem auf den Klimaschutz. Die Ausweitung vom Zielpaar zur Zieltrias vergrößert zugleich die Herausforderung, Zielkonflikten zu begegnen und Prioritäten zu setzen. ${ }^{6}$

\section{Die Beschlüsse des Europäischen Rates}

Der Europäische Rat verabschiedete auf seinem Treffen im März 2007 in Brüssel den Aktionsplan „Eine Energiepolitik für Europa“ für die Jahre 2007 bis 2009.7 Er ist in mehrere Themenbereiche untergliedert. An erster Stelle steht die Vollendung des Erdgas- und Elektrizitätsbinnenmarktes; angestrebt werden eine vollständige Umsetzung der bestehenden Richtlinien sowie eine ,wirksame Trennung der Netze von Versorgung und Erzeugung“ und eine Harmonisierung der Befugnisse der nationalen Regulierungsstellen. Eine ,tatsächliche Diversifizierung der Energiequellen und Transportrouten“ soll die Versorgungssicherheit erhöhen. In diesem Zusammenhang wird auch vorgeschlagen, ein gemeinsames Konzept für eine Energieaußenpolitik zu entwickeln. In den Medien am stärksten wahrgenommen wurden drei 20-Prozent-Ziele: Zum einen sollen 20 Prozent des EU-Energieverbrauchs gemessen an

3 Daniel Göler/Mathias Jopp: Kann Europa gelingen? Vorhaben und Chancen der deutschen Ratspräsidentschaft, in: integration 1/2007, S. 8.

4 Vgl. Svein S. Andersen: Energy policy: Interest interaction and supranational authority, in: Svein S. Andersen/ Kjell A. Eliassen (Hrsg.): Making Policy in Europe, London 2001, S. 106-123.

5 Vgl. Francis McGowan: European Energy Policies in a Changing Environment, Heidelberg 1996.

6 Vgl. Francis McGowan: Conflicting Objectives in European Energy Policy, in: Colin Crouch/David Marquand (Hrsg.): The Politics of 1992: Beyond the Single Market, Oxford 2001, S. 121-137.

7 Europäischer Rat (Brüssel): Schlussfolgerungen des Vorsitzes, 8./9. März 2007, abrufbar unter: http://register.consilium.europa.eu/pdf/de/07/st07/st07224.de07.pdf (letzter Zugriff: 21.6.07). 
den Prognosen für 2020 eingespart sowie dann 20 Prozent des Primärenergieverbrauchs durch erneuerbare Energien gedeckt werden. Die Umsetzung der im Aktionsplan beschlossenen Maßnahmen wird durch den Europäischen Rat einer jährlichen Überprüfung unterzogen werden. Daneben einigte sich der Europäische Rat in den Schlussfolgerungen darauf, in der Europäischen Union bis 2020 die Treibhausgasemissionen um 20 Prozent im Vergleich zu $1990 \mathrm{zu}$ vermindern. Im Hinblick auf die Verhandlungen über ein Folgeabkommen für das im Jahr 2012 auslaufende Kyoto-Protokoll zur Reduzierung der Treibhausgase stellt die Europäische Union in Aussicht, das 20-Prozent-Ziel auf 30 Prozent anzuheben, sofern sich andere Industrieländer auch dazu verpflichten. Mit diesem als ehrgeizig eingestuften Ziel und der angestrebten engen Verzahnung der Energie- und Klimaschutzpolitik will der Europäische Rat die Vorreiterrolle Europas im Klimaschutz unterstreichen und wurde in diesem Bestreben stark vom Europäischen Parlament unterstützt. ${ }^{8}$ Die Gipfelbeschlüsse wurden auch als klare Herausforderung an die USA sowie China und Indien aufgefasst. ${ }^{9}$

\section{Der weite Weg zum Energiebinnenmarkt}

Die Einführung von Wettbewerb auf den europäischen Märkten für Strom und Gas und deren Zusammenwachsen zu einem gemeinsamen Markt verfolgt das Ziel, sichere Energie zu wettbewerbsfähigen Preisen für die europäischen Volkswirtschaften zu gewährleisten. In ihrem Energiepaket vom Januar 2007 betont die Kommission zudem mit Blick auf den Emissionshandel sowie den Netzanschluss neuer dezentraler Stromerzeugungskapazitäten, dass der Binnenmarkt auch erheblich zur Nachhaltigkeit beitragen werde. ${ }^{10}$

Die ersten Liberalisierungsrichtlinien Strom ${ }^{11}$ (1996) und Gas ${ }^{12}$ (1998) sollten für zumindest größere Stromverbraucher zu freiem Wettbewerb führen. ${ }^{13}$ Allerdings blieben die Entwicklungen auf den europäischen Märkten weit hinter den Erwartungen zurück - zu beobachten waren eine teils sehr geringe Marktöffnung, Intransparenz, hohe Marktdominanz der etablierten und Diskriminierung neuer Anbieter sowie mangelnde Infrastrukturinvestitionen. ${ }^{14}$

Die Beschleunigungsrichtlinien Strom ${ }^{15}$ und Gas ${ }^{16}$ (2003) sollten diesen Mängeln begegnen und die Liberalisierung und Integration der europäischen Energiemärkte vollenden. Vorgegeben wurde die vollständige Marktöffnung für alle Kundengruppen bis 2007 (Ausnahmen bestehen für neuere Beitrittsländer), die Regulierung des Netzzugangs und der Netznutzung, damit in Zusammenhang die Einrichtung einer Regulierungsbehörde für die Aufsicht über Netzzugangs- und -nutzungskonditionen sowie eine rechtliche Entflechtung des Netzbetriebs von Erzeugung und Absatz.

Unter anderem aufgrund steigender Energiepreise und anhaltender Beschwerden leitete die Kommission im Sommer 2005 eine Sektoruntersuchung ${ }^{17}$ der Strom- und Gasmärkte

8 Vgl. Europäisches Parlament: Beitrag zur Frühjahrstagung 2007 des Europäischen Rates im Hinblick auf die Lissabon-Strategie, Entschließung B6 0043/2007, 7.02.2007.

9 Vgl. etwa New York Times: Europe Sets Ambitious Limits on Greenhouse Gases, 10.03.2007.

10 Europäische Kommission: Eine Energiepolitik für Europa, 2007, S. 7.

11 Richtlinie 96/92/EC, in: Amtsblatt, Nr. L 27/1997, S. 20-29.

12 Richtlinie 98/30/EC, in: Amtsblatt, Nr. L 204/1998, S. 1-12.

13 Vgl. Rainer Eising: Die regulative Reform der Elektrizitätsversorgung in Großbritannien, der Europäischen Gemeinschaft und der Bundesrepublik, Opladen 2000.

14 Vgl. Europäische Kommission: Zweiter Benchmarkingbericht über die Vollendung des Elektrizitäts- und Erdgasbinnenmarktes, SEK(2002) 1038 1, Oktober 2002.

15 Richtlinie 2003/54/EC, in: Amtsblatt, Nr. L 176/2003, S. 37-56.

16 Richtlinie 2003/55/EC, in: Amtsblatt, Nr. L 176/2003, S. 57-78.

17 Die Generaldirektion Wettbewerb der Kommission hat die Möglichkeit, eine Sektoruntersuchung durchzuführen, wenn Zweifel über das Funktionieren des Wettbewerbs in einem Sektor bestehen, ohne dass die Ursache hierfür erkenntlich ist. Die Ergebnisse können darauf als Grundlage für wettbewerbsrechtliche Verfahren dienen. 
ein. Der im Januar 2007 vorgelegte Abschlussbericht ${ }^{18}$ konstatiert erhebliche Unzulänglichkeiten des Wettbewerbs. So bestehe auf den meisten nationalen Märkten eine zu hohe Anbieterkonzentration in der Erzeugung, ${ }^{19}$ der Eintritt neuer Marktakteure werde behindert, die Märkte der Mitgliedstaaten seien weiterhin untereinander zu wenig verbunden und transparente Marktinformationen nicht ausreichend verfügbar. Auch regulieren (,deckeln') einige Länder, etwa Frankreich und Polen, die Endkundenpreise, wodurch der Eintritt neuer Marktteilnehmer sowie Investitionen in zusätzliche Kapazitäten behindert werden. Behinderungen für neue Anbieter bestehen auch durch Langzeit-Lieferverträge, die häufig für Gas, jedoch auch für Strom abgeschlossen werden. Schließlich haben die Regulierungsbehörden, die die diskriminierungsfreie Nutzung der Energienetze sicherstellen sollen, sehr unterschiedliche Kompetenzen und müssen teils politischen Vorgaben folgen. ${ }^{20}$ Die zyprische Behörde sowie die deutsche Bundesnetzagentur ${ }^{21}$ etwa haben erst jüngst ihre Arbeit aufgenommen und es lassen sich lediglich anfängliche Auswirkungen auf dem Energiemarkt beobachten. Die Vorgaben zur Entflechtung wurden häufig formal umgesetzt, dennoch bleiben teils erhebliche Bedenken hinsichtlich diskriminierenden Verhaltens durch die etablierten Versorger. Obgleich in vielen Fällen einige Übertragungskapazitäten für Strom zwischen den Mitgliedstaaten bestehen, sind diese oft überlastet. Daher hält die Kommission ein Maßnahmenbündel für erforderlich, um ,innerhalb von drei Jahren ein europäisches Gas- und Stromnetz sowie einen wirklich wettbewerbsorientierten europaweiten Energiemarkt zu schaffen“. ${ }^{22}$

Die Beschlüsse des Europäischen Rates sind deutlich zurückhaltender, auch wird die Nennung eines Zeitpunktes für die Vollendung des Binnenmarktes vermieden - nach dem zweiten Liberalisierungspaket sollte die Marktöffnung für alle Verbraucher ab Sommer 2007 gelten. Zentral ist für die Kommission derzeit eine eigentumsrechtliche Entflechtung des Netzbetriebs von Erzeugung und Versorgung. Dies wird von Großbritannien stark befürwortet, dagegen von Frankreich und Deutschland strikt abgelehnt, ${ }^{23}$ und der Europäische Rat konnte sich im März nur auf eine „,wirksame Entflechtung“ und ,unabhängig organisierte Strukturen“ einigen. Die Generaldirektionen Energie und Wettbewerb verfolgen dennoch weiterhin eine eigentumsrechtliche Entflechtung der Unternehmen (in der Presse vielfach als „Zerschlagung“ bezeichnet) und im September soll ein entsprechendes Richtlinienpaket vorgeschlagen werden; nach dem Wunsch von Energiekommissar Andris Piebalgs das letzte zur EnergiemarktLiberalisierung. ${ }^{24}$ Anfang Juni entstand auf dem Treffen der europäischen Energieminister der Eindruck, eine Mehrheit der Mitgliedsländer lehne eine Entflechtung ab. Mindestens acht Regierungen unterstützen die Kommission in dieser Hinsicht allerdings weiterhin aktiv. ${ }^{25}$

Neben dem legislativen Weg besteht nach Artikel 7 VO 1/2003 auch die Möglichkeit, dass die Kommission im Rahmen von Kartellverfahren eine Abspaltung der Netze verfügt. ${ }^{26}$

18 Vgl. Europäische Kommmission: DG Competition Report on Energy Sector Inquiry, Brussels 2007.

19 Vgl. auch Felix Chr. Matthes/Katherina Grashof/Sabine Gores: Power Generation Market Concentration in Europe 1996-2005. An Empirical Analysis, Berlin 2007.

20 Vgl. Christophe Genoud/Matthias Finger: Electricity regulation in Europe, in: Dominique Finon/Atle Midttun (Hrsg.): Reshaping European Gas and Electricity Industries: Regulation, Markets, and Business Strategies, Amsterdam 2004, S. 29-68.

21 Ausführlich zur Einrichtung der Bundesnetzagentur und ihrer Kompetenzausstattung vgl. Katherina Grashof: „Wollen wir wirklich so einen strengen Regulierer?“" Regulierung von Stromnetzentgelten, Discussion Paper Nr. 2007-003, Berlin 2007.

22 Vgl. Europäische Kommission: Eine Energiepolitik für Europa, 2007, S. 8-12.

23 Vgl. Handelsblatt: EU gibt Entwarnung für Energiekonzerne, 16.02.2007.

24 Vgl. Handelsblatt: Brüssel will Versorger zerschlagen, 23.04.2007 und Andris Piebalgs: Hin zu einem Europäischen Binnenmarkt für Strom und Gas, Rede auf dem VDEW-Kongress 2007 am 24.05.2007.

25 Vgl. Financial Times Deutschland: EU-Staaten gehen gegen Strommultis an, 26.06.2007.

26 Verordnung (EG) Nr. 1/2003 des Rates vom 16. Dezember 2002 zur Durchführung der in den Artikeln 81 und 82 des Vertrags niedergelegten Wettbewerbsregeln, in: Amtsblatt, Nr. L 001/2003, S. 1-25. 
Weiter befürwortet der Europäische Rat stärkere (Kooperations-)Kompetenzen der Regulierungsbehörden, eine Verbesserung des grenzüberschreitenden Handels und Netzbetriebs sowie der Einbindung neuer Kraftwerke ins Stromnetz.

Als Teil des Energiepakets hatte die Kommission im Januar 2007 zur Stärkung des Binnenmarktes einen vorrangigen Verbundplan vorgelegt. Hier wird - auch dies unterstützt der Europäische Rat ausdrücklich - die Ernennung von vier europäischen Koordinatoren vorgeschlagen, die vier prioritäre Projekte des Netzausbaus beschleunigen sollen. ${ }^{27}$ Auch das Europäische Parlament sieht hierin wichtige Schritte zur Erhöhung des Verbundgrades der europäischen Stromnetze; es begrüßt zudem ausdrücklich das Vorgehen der Kommission gegen Mitgliedstaaten, ,die in unzulässiger Weise nationale Energieriesen schützen““28

Denn trotz der wiederholten einmütigen Bekenntnisse der Regierungen zum europäischen Binnenmarkt fallen derzeit eher protektionistische Tendenzen auf, die zugleich wesentliche Zielkonflikte zwischen Wettbewerb und Versorgungssicherheit verdeutlichen. Beispielhaft zeigte sich dies am Widerstand der spanischen Regierung gegen die Übernahme Endesas durch den deutschen Versorger Eon sowie der Rolle Italiens; in Frankreich ist Ähnliches zu beobachten. ${ }^{29}$ Die Kommission ist zwar gegen die spanische Regierung vorgegangen, hat das Scheitern der Übernahme jedoch nicht verhindern können. Viele nationale Regierungen sehen, ihre" etablierten Energieversorger als mit Blick auf die europäischen und globalen Energiemärkte strategisch wichtige Unternehmen an, deren Interessen durch zu intensiven Wettbewerb im Inland und mit dem Ausland nicht geschadet werden dürfe. Einerseits erfüllt die wachsende Anzahl und Größe von Unternehmensfusionen im europäischen Energiesektor viele Regierungen offenbar mit Sorge, dass ,eigene' Energieversorger von ausländischen Unternehmen übernommen oder zumindest nicht im Kreis der am Ende der Fusionswelle erwarteten wenigen, europäischen Champions' sein werden. Andererseits gilt dies im Hinblick auf den Zugang zu Öl und Gas - die einen Länder haben eigene rohstofffördernde Unternehmen (etwa Frankreich und Großbritannien), die anderen (etwa Deutschland) hoffen durch die Größe ihrer Importeure auf eine gute Verhandlungssituation.

\section{Diversifizierung der Rohstoffbezugsquellen und Transportrouten - die Sorge vor Russlands Hand am Gashahn}

Die zunehmende Abhängigkeit von importiertem Öl und vor allem Erdgas wird in der gegenwärtigen Debatte oft als zentrales Problem für die zukünftige Energieversorgung Europas dargestellt - angesichts eines möglichen Anstiegs der Importabhängigkeit der Europäischen Union am Gesamtenergieverbrauch von heute 50 Prozent auf 65 Prozent im Jahr 2030 (Erdgas: von 57 Prozent auf 84 Prozent; Erdöl: von 82 Prozent auf 93 Prozent).$^{30}$ Derzeit bezieht die Europäische Union die Hälfte ihrer Erdgaseinfuhren aus drei Ländern: Russland, Norwegen und Algerien.

Im Bereich der Energieversorgungssicherheit sind bis heute im Wesentlichen zwei Richtlinien aus den 1970er Jahren relevant: zum einen eine Richtlinie (1968) zur Mindestvorratshaltung von Rohöl zur Überbrückung von Versorgungslücken für die Dauer von 90 Tagen, ${ }^{31}$

27 Europäische Kommission: Mitteilung an den Rat und das Europäische Parlament, KOM(2006) 846 endgültig, S. 12.

28 Europäisches Parlament: Beitrag zur Frühjahrstagung 2007, Punkt 75.

29 Vgl. Enno Harks/Andreas Pointvogl: Deutschlands Energieversorgungssicherheit im Schatten europäischer Energiemärkte, SWP- Diskussionspapier FG 8, 2007/Nr. 5, April 2007, Berlin.

30 Vgl. Europäische Kommission: Eine Energiepolitik für Europa, 2007, S. 4.

31 Richtlinie 68/414/EWG, in: Amtsblatt, Nr. L 308/1968, S. 14-16 sowie Richtlinie 72/425/EWG, in: Amtsblatt, Nr. L 291/1972, S. 154. 
zum anderen eine Richtlinie (1973) zur Aufstellung von nationalen Kriseninterventionsplänen im Falle eines Erdölversorgungsengpasses ${ }^{32}$ Diese Vorkehrungen werden in enger Abstimmung mit der Internationalen Energie-Agentur umgesetzt. Ähnliche Vorgaben für den Erdgasbereich bestehen nicht.

Bereits seit den 1970er Jahren hat Europa außerdem den verstärkten Dialog mit Lieferantenländern gesucht, der in den letzten Jahren institutionalisiert und ausgeweitet wurde, beispielsweise im Herbst 2005 in Form der Europäischen Energiegemeinschaft. Damit wird der Energiebinnenmarkt praktisch auf die gesamte Balkanhalbinsel ausgedehnt. Strategisch wird so ein neuer Versorgungsweg für Erdgas aus dem Nahen Osten und der Region des Kaspischen Meers erschlossen. ${ }^{33}$ Ein weiteres wichtiges vertragliches Instrument ist die Energiecharta (1998 in Kraft getreten), die zum Ziel hat, die multilaterale Zusammenarbeit im Energiesektor nach Regeln der Welthandelsorganisation zu verstärken. Durch den vom Transitprotokoll als Teil der Energiecharta garantierten freien Transitzugang zu den Vertragsstaaten sieht Russland seine Monopolstellung als Transit- und damit Lieferland für die europäische Energieversorgung aus dem Osten in Frage gestellt und verweigert daher die Ratifizierung. ${ }^{34}$ Stattdessen wurden die Beziehungen zwischen der Europäischen Union und Russland im Rahmen des Partnerschafts- und Kooperationsabkommens 1997 institutionalisiert, das jedoch Ende 2007 ausläuft und dessen Verlängerung derzeit zentrales Thema zwischen Russland und der Europäischen Union ist. Die schwierigen Verhandlungen mit Russland führen dazu, dass der energiepolitische Dialog mit anderen Regionen immer wichtiger wird. So wurde 2004 die sogenannte ,Baku-Initiative“ mit den Staaten am Kaspischen Meer ins Leben gerufen, die im November 2006 zu einer ,Energy Road Map ‘ührte und eine engere Kooperation im Energiebereich anstrebt. ${ }^{35}$ Auch das Energieforum der Partnerschaft Europa-Mittelmeer verfolgt ähnliche Ziele. ${ }^{36}$ Außerdem fand im Juni 2005 ein erstes bilaterales Treffen zwischen der Europäischen Union und der Organization of the Petroleum Exporting Countries (OPEC) statt.

Trotz eines gemeinsamen Interesses an Energieversorgungssicherheit zeigt sich aber nach wie vor eine heterogene Interessenslage der Mitgliedstaaten. Dies lässt sich an zwei Beispielen verdeutlichen: zum einen an der Rolle bestimmter Energieträger am nationalen Energiemix, zum anderen am Verhältnis zu Energielieferländern. Während zum Beispiel Frankreich aufgrund hoher Kernenergie-Stromerzeugung relativ wenig als unsicher erachtete fossile Energie importiert, bestehen in den osteuropäischen Staaten hohe Gasimport-Anteile - in erster Linie in den baltischen Staaten, wo nahezu die gesamten Gasimporte aus Russland stammen. Darüber hinaus verfügen nur wenige Mitgliedsländer wie Deutschland über eine systematische Gasvorratshaltung.

Was die strategische Positionierung einzelner Mitgliedstaaten gegenüber Energielieferländern angeht, ist der Position gegenüber Russland zentrale Bedeutung beizumessen. Aufgrund der ,special relationship“37 zwischen Deutschland und Russland scheint für Deutsch-

32 Richtlinie 73/238/EWG, in: Amtsblatt, Nr. L 228/1973, S. 1-2.

33 Vgl. „Die EU und Südosteuropa unterzeichnen einen historischen Vertrag zur Förderung der Energieintegration“, Pressemitteilung, IP/05/1346, 25.10.2005.

34 Vgl. Kirsten Westphal: Energy Policy between Multilateral Governance and Geopolitics: Whither Europe?, in: Internationale Politik und Gesellschaft 4/2006, S. 53-55.

35 Vgl. „The EU and the countries of the Black Sea and Caspian Sea regions agree on a common energy strategy", Pressemitteilung, IP/06/1657, 30.11.2006.

36 Vgl. Europäische Kommission: Mitteilung an den Rat und das Europäische Parlament über die Vorbereitung der Tagung der Außenminister der Partnerschaft Europa-Mittelmeer in Tampere (27./28. November 2006): Die Partnerschaft Europa-Mittelmeer: Zeit zum Handeln, KOM(2006) 620 endgültig, S. 8-9.

37 Vgl. Dieter Helm: European energy policy: meeting the security of supply and climate change challenges, draft January 2007, to be published in: European Investment Bank Papers 2007, abrufbar unter: http://www.dieterhelm.co.uk/publications/EIB_paper.pdf (letzter Zugriff: 22.5.07), S. 15. 
land entgegen häufig vertretenen Forderungen eine Reduzierung der Importabhängigkeit von Russland nicht an erster Stelle zu stehen, wie sich am Bau der Ostseepipeline zwischen den beiden Ländern zeigt. Für die baltischen Staaten bieten sich als Option zur Diversifizierung weg von Russland Flüssiggas-Terminals an, um verflüssigtes Gas per Schiff zu importieren, was allerdings mit hohen Kosten verbunden ist. Die geplante Nabucco-Pipeline zum Transport von Gas aus der Kaspischen Region von der Türkei nach Mitteleuropa wurde daher vom ungarischen Premierminister Gyurcsany als ,langer Traum“ "bezeichnet. ${ }^{38}$

Angesichts dieser Ausgangssituation fordert der Aktionsplan eine Diversifizierung der Energiequellen und Transportrouten durch die Fortführung bereits bestehender Initiativen (zum Beispiel Energiegemeinschaft) und sieht darin auch einen Beitrag zur Erhöhung des Wettbewerbs im europäischen Energiebinnenmarkt. Außerdem wird das Prinzip der Solidarität zwischen den Mitgliedstaaten betont. Das Fehlen gemeinschaftlicher Maßnahmen im Erdgasbereich (beispielsweise gemeinschaftliche Vorratshaltung wie im Ölbereich) wird im Aktionsplan jedoch nicht explizit behandelt; stattdessen werden lediglich Prüfaufträge vergeben.

Die tatsächliche Bedeutung des Solidaritätsprinzips spiegelt sich jedoch im Aktionsplan dort wider, wo von ,der vorrangigen Verantwortung der Mitgliedstaaten, für ihren heimischen Bedarf Rechnung zu tragen", 39 die Rede ist. Während die geschlossene Haltung der Europäischen Union gegenüber Russland anlässlich des Gipfels im russischen Samara im Mai 2007 als Solidaritätsbeweis zwischen den Mitgliedstaaten gewertet werden kann, ${ }^{40}$ stellt sich im Hinblick auf die Umsetzung der Beschlüsse die Frage, ob alle Mitgliedstaaten eine gemeinschaftliche Energieaußenpolitik tatsächlich unterstützen werden (Beispiel Ostseepipeline). Zwar sind sich Mitgliedstaaten wie Frankreich, Polen, Deutschland und Großbritannien in Kernpunkten offiziell einig (Beispiel Diversifizierung der Bezugsquellen), doch bestehen unterschiedliche strategische Bewertungen. ${ }^{41} \mathrm{Ob}$ ein ,représentant spécial pour l'énergie“, 42 wie vom französischen Premier de Villepin 2006 vorgeschlagen, zur Vergemeinschaftung beitragen könnte, ist zu bezweifeln, solange die Mitgliedstaaten hier weiterhin über ein Veto verfügen. So begegnete Ungarn Vorwürfen, es würde mit der Unterstützung einer russischen Gaspipeline (,Blue Stream') aus der Türkei nach Ungarn die gemeinsame europäische Energiepolitik in Frage stellen, lapidar, dass man nicht in Frage stellen könne, was es gar nicht gäbe. ${ }^{43}$

Trotz Diversifizierung kann jedoch die Abhängigkeit von Russland kaum völlig beseitigt werden; ebenso wenig wie die Gefahr physischer Versorgungsstörungen durch Leitungsdefekte oder -manipulationen. Mit der Ausklammerung der strategischen Gasvorratshaltung ist der Europäische Rat dieser Frage aus dem Weg gegangen. Während kommerzielle Gasvorräte im Interesse der Gasversorger sind, um die Erfüllung ihrer Vertragsverpflichtungen zu gewährleisten, sind Energieunternehmen gegenüber strategischen Vorratshaltungen skeptisch, da diese tendenziell Preise und damit Gewinne senken. ${ }^{44}$ Es zeichnet sich ab, dass die

38 Vgl. The Economist: Europe: A bear at the throat, European energy security, 14.04.07.

39 Europäischer Rat: Schlussfolgerungen des Vorsitzes, 8./9. März 2007, S. 18.

40 Vgl. z.B. Catherine Belton/Stefan Wagstyl: EU members stand with Poland to resist Kremlin, in: Financial Times, 19.05.2007.

41 Vgl. Oliver Geden/Clémence Marcelis/Andreas Maurer: Perspectives for the European Union's External Energy Policy: Discourse, Ideas and Interests in Germany, the UK, Poland and France, SWP Working Paper FG 1, 17.12.2006.

42 Vgl. Allocution du Premier ministre au 10e Forum international de la fondation Bertelsmann, abrufbar unter: http://www.premier-ministre.gouv.fr/acteurs/interventions_premier_ministre_9/discours_498/ allocution_10e_forum_international_56920.html (letzter Zugriff: 25.09.2006).

43 Vgl. The Economist: Europe: A bear at the throat, European energy security, 2007.

44 Helm: European energy policy, 2007, S. 25-26. 
Internationale Energie-Agentur im Bereich der Gasvorratshaltung nun eine Rolle übernehmen könnte, zu der die Europäische Union nicht in der Lage scheint. ${ }^{45}$

\section{Energieeffizienz - alles nur Symbolpolitik?}

Energieeffizienz bezieht sich sowohl auf die Erzeugung wie die Nutzung von Strom und Wärme. Einerseits ist etwa die Nutzung der Abwärme bei der Stromerzeugung (KraftWärme-Kopplung) gemeint und damit eine effizientere Ausnutzung des Brennstoffes sowie andererseits eine Reduktion des Endenergieverbrauchs durch sparsamere Geräte, Motoren oder Industrieprozesse. Obgleich eine Verbesserung der Energieeffizienz für Versorgungssicherheit, Wettbewerbsfähigkeit wie für Klimaschutz ein außerordentlich effektives Instrument darstellt, rangiert es in der öffentlichen Debatte häufig unter ,ferner liefen", und politikinduzierte Forschritte sind kaum zu beobachten. Dies mag an der häufig notwendigen Vielzahl der Akteure liegen, die zu mobilisieren wären und auch an einem Interesse von Energieversorgern, ihren Absatz nicht zu verringern.

Energieeffizienz und die Reduzierung der Energienachfrage wurden in Brüssel bereits in den 1970er Jahren infolge der ersten Ölkrise als wichtiger Beitrag zur Energieversorgungssicherheit identifiziert. ${ }^{46}$ Inzwischen wird vor allem ihr potenzieller Beitrag zur Verbesserung der Wettbewerbsfähigkeit der europäischen Volkswirtschaften betont. Die Kommission geht davon aus, dass 20 Prozent des derzeitigen Energieverbrauchs in der Europäischen Union und damit jährlich 100 Milliarden Euro eingespart werden könnten. ${ }^{47}$

Energieeffizienzrichtlinien wurden seit Anfang der 1990er Jahre verabschiedet und betreffen die Kennzeichnung von Haushaltsgeräten ${ }^{48}$ die Herstellungsplanung von Endgeräten, ${ }^{49}$ den Gebäudeenergieverbrauch und Energiedienstleistungen. ${ }^{50}$ Allerdings besteht bei den Mitgliedsländern ein hohes Umsetzungsdefizit. So wurde die Richtlinie zur Gesamtenergieeffizienz von Gebäuden von nur 16 Mitgliedstaaten fristgerecht umgesetzt, während die meisten Mitgliedstaaten beschlossen haben, wesentliche Teile der Richtlinie verspätet umzusetzen. ${ }^{51}$ Der Energieeffizienzaktionsplan der Kommission wurde vom Europaabgeordneten Chris Davies daher als „Frustrationsschrei“"52 bezeichnet.

Mit diesem im Oktober 2006 vorgelegten Energieeffizienzaktionsplan mit 70 Einzelmaßnahmen unterstreicht die Kommission ihre Ambitionen in diesem Bereich.53 Allerdings werden darin an keiner Stelle die (Miss-)Erfolge ihres Aktionsplanes aus dem Jahre 2000 analysiert. Der Erfolg hängt jedoch entscheidend vom erhöhten Engagement der Mitgliedstaaten ab. Während der Ministerrat den Energieeffizienzaktionsplan der Kommission begrüßte, hebt er in seiner Stellungnahme, ,unter den zahlreichen guten Vorschlägen des Aktionsplans“ lediglich fünf vorrangige Maßnahmen hervor. ${ }^{54}$ Unter diesen Maßnahmen bezieht

45 Vgl. Gesche Wüpper: Energie-Agentur dringt auf Gasreserven für Notfälle, in: Die Welt, 15.05.2007, S. 11.

46 Vgl. Towards a new energy policy strategy for the Community. Communication presented to the Council by the Commission on 5 June 1974, in: Bulletin of the European Communities, Supplement 4/74.

47 Vgl. Europäische Kommission: Eine Energiepolitik für Europa, 2007.

48 Richtlinie 92/75/EWG, in: Amtsblatt, Nr. L 297/1992, S. 16-19.

49 Richtlinie 2005/32/EG, in: Amtsblatt, Nr. L 191/2005, S. 29-58.

50 Richtlinie 2002/91/EG, in: Amtsblatt, Nr. L 1/2003, S. 65-71 und Richtlinie 2006/32/EG, in: Amtsblatt, Nr. L 114/2006, S. 64-85.

51 Vgl. European Climate Change Programme: Report from EPPC Energy Demand meetings 25 January, 23 February and 2 May 2006, abrufbar unter: http://circa.europa.eu/Public/irc/env/eccp_2/library?l=/eccp_review_ energy_1/final18jul06doc/_EN_1.0_\&a=d (letzter Zugriff: 20.5.07).

52 ENDS Europe Daily: Commission sets out EU energy efficiency vision, Issue 2188, 16.10.2006.

53 Vgl. Europäische Kommission: Aktionsplan für Energieeffizienz: Das Potenzial ausschöpfen, KOM(2006) 545 endgültig, 19.10.2006.

54 Rat der Europäischen Union: Annahme von Schlussfolgerungen des Rates zum Aktionsplan für Energieeffizienz, 15210/06, 16.11.06, S. 8. 
sich jedoch keine auf die Vorschläge der Kommission zur ,,verbesserte[n] Energieumwandlung“", die vor allem auf eine Erhöhung des Kraft-Wärme-Kopplung-Anteils und der dezentralen Stromerzeugung im Allgemeinen abzielten. Umweltverbände äußerten die Vermutung, dass der Energieeffizienzaktionsplan der Kommission auf Druck der Energiewirtschaft in letzter Minute noch verwässert wurde. ${ }^{55}$ Die Ausklammerung kann als im Sinne der etablierten Energieunternehmen interpretiert werden, die im Klimaschutz bevorzugt auf großtechnologische Lösungen wie ,saubere Kohle‘ oder Kernenergie setzen.

Die Gipfelbeschlüsse berufen sich hauptsächlich auf die vom Ministerrat im November 2006 verabschiedeten ,,vorrangigen Maßnahmen“. Dies kann als Scheitern der europäischen Energiepolitik in diesem Bereich interpretiert werden; neben dem bisher vernachlässigten Verkehrsbereich und Verbraucherverhalten wird ,lediglich' die Umsetzung oder der Ausbau bereits bestehender Richtlinien angestrebt. Das Erreichen des in den Medien so stark wahrgenommenen, unverbindlichen 20-Prozent-Ziels ist mit der weitgehenden Ausklammerung des Energieumwandlungssektors, der bis 2020 über ein Drittel der Gesamtenergieeinsparung erbringen sollte, mehr als ungewiss. ${ }^{56}$ Die Festlegung eines unverbindlichen Ziels in diesem Bereich ist vermutlich auch darauf zurückzuführen, dass die Umsetzung von Energieeffizienzmaßnahmen die Einbindung einer Vielzahl von Akteuren erfordert und damit ein oft schwieriger Prozess ist. ${ }^{57}$

\section{Erneuerbare Energien - der große Durchbruch?}

Auf europäischer Ebene gewann dieses Thema Mitte der 1990er Jahre an Bedeutung. Das 1997 vorgelegte Weißbuch forderte einen Anteil von zwölf Prozent erneuerbarer Energieträger am Primärenergieverbrauch bis $2010 .{ }^{58}$ Der Anteil der erneuerbaren Energieträger am Primärenergieverbrauch unterscheidet sich beträchtlich zwischen den Mitgliedstaaten: 2004 betrug der durchschnittliche Anteil für die EU-25 6,3 Prozent (von 1,6 Prozent im Vereinigten Königreich und Luxemburg bis zu 35,9 Prozent in Lettland). ${ }^{59}$

Derzeit sind zwei Richtlinien zur Förderung erneuerbarer Energien in Kraft. Die Richtlinie zur Förderung der Stromerzeugung aus erneuerbaren Energien (2001) sieht bis zum Jahr 2010 einen Anteil von 21 Prozent am Stromverbrauch vor ${ }^{60}$ Für jeden Mitgliedstaat bestehen unverbindliche Ziele, auch die Auswahl der Förderinstrumente ist freigestellt; alle zwei Jahre bewertet die Kommission die Zielerreichung. Die Biokraftstoff-Richtlinie verlangt ebenfalls unverbindlich eine Erhöhung des Anteils von Biokraftstoffen in der Europäischen Union auf 5,75 Prozent bis $2010 .{ }^{61}$ Der gegenwärtige Fortschritt bei der Erhöhung erneuerbarer Energien in der Europäischen Union wird von einigen wenigen Ländern wie Deutsch-

55 Vgl. Euractiv.com: Energy efficiency: action plan ditched or strengthened?, abrufbar unter: http://www.euractiv.com/en/energy/energy-efficiency-action-plan-ditched-strengthened/article-158224 (letzter Zugriff: 31.5.07).

56 Vgl. Europäische Kommission: Eine europäische Strategie für nachhaltige, wettbewerbsfähige und sichere Energie, 2006, S. 32.

57 Vgl. Europäische Kommission: Weniger kann mehr sein. Grünbuch über Energieeffizienz, KOM(2005) 265 endgültig, 22.06.2005, S. 15.

58 Vgl. Mitteilung der Europäischen Kommission: Energie für die Zukunft: erneuerbare Energieträger, KOM(97) 599 endgültig.

59 Vgl. Bundesministerium für Umwelt, Naturschutz und Reaktorsicherheit: Erneuerbare Energien in Zahlen nationale und internationale Entwicklung - Stand: Januar 2007 - Internet-Update, abrufbar unter: http:// www.erneuerbare-energien.de/files/erneuerbare_energien/downloads/application/pdf/ broschuere_ee_zahlen.pdf (letzter Zugriff: 19.5.07).

60 Richtlinie 2001/77/EG, in: Amtsblatt, Nr. L 283/2001, S. 33-40.

61 Richtlinie 2003/30/EG, in: Amtsblatt, Nr. L 123/2003, S. 42-46. 
land, Spanien und Dänemark getragen, ${ }^{62}$ die Gesamtzielerreichung für 2010 erscheint jedoch sehr unwahrscheinlich. ${ }^{63}$

Die Interessen und Strategien der Mitgliedsländer in Bezug auf die Förderung erneuerbarer Energien wurden auf dem Gipfeltreffen vor allem vom bestehenden Energiemix und Erwartungen über die Kostenbelastungen bestimmt. Für Frankreich war ein verbindlicher Anteil erneuerbarer Energieträger am europäischen Energiemix 2020 nur akzeptabel, wenn bei der Zielformulierung für die einzelnen Länder die Kernenergie als $\mathrm{CO}_{2}$-arme Energiequelle berücksichtigt wird. ${ }^{64}$ Diese Position wurde vor allem von der tschechischen Republik geteilt. Dagegen haben atomkritische Länder wie Dänemark, Irland und Österreich eine solche Verrechnung abgelehnt. Viele osteuropäische Länder befürchten insbesondere hohe Kosten durch ehrgeizige Ziele. Vor allem in Polen wird die Nutzung vergleichsweise günstiger heimischer Kohle bevorzugt. Deutschland, Spanien und Dänemark waren starke Befürworter eines verbindlichen Ziels, nicht zuletzt aufgrund der gut entwickelten heimischen Industrien in diesem Bereich.

Innerhalb der Kommission ist die energiepolitische Vorgehensweise im Bereich der erneuerbaren Energiepolitik umstritten. Vor allem die Generaldirektion Wettbewerb lehnt nationale Einspeisevergütungen, die sich als das effektivste Instrument erwiesen haben, ${ }^{65}$ nach wie vor ab und sieht darin illegale staatliche Beihilfen, wie sich jüngst in ihrer Kritik an den luxemburgischen und slowenischen Einspeisegesetzen gezeigt hat. ${ }^{66}$ Das Europäische Parlament ist dagegen ein wichtiger Partner für die Befürworter der erneuerbaren Energien innerhalb der Kommission. Es hat in seinem Bericht zum Grünbuch im Dezember 2006 noch ehrgeizigere Ziele gefordert (25 Prozent am Primärenergieverbrauch für erneuerbare Energien bis 2020) und vor allem auf verbindliche sektorale Zielvorgaben gedrängt. ${ }^{67}$ Der europäische Industrieverband BusinessEurope lehnt ein bindendes 20-Prozent-Ziel bis 2020 ab und fordert die Harmonisierung der nationalen Förderinstrumente (jedoch nicht hin zu Einspeisevergütungen), um einen kosteneffizienten Ausbau zu gewährleisten. Eine ähnliche Position vertritt die europäische Stromindustrie. ${ }^{68}$

Angesichts der Tatsache, dass das bestehende 2010-Ziel von zwölf Prozent aufgrund unzureichender Anstrengungen in der Mehrzahl der Mitgliedstaaten wohl nicht mehr zu erreichen ist, ${ }^{69}$ stellt sich die Frage, inwieweit das Erreichen des 20-Prozent-Ziels bis 2020 realistisch ist. Bereits 2004 hat die Kommission neue Maßnahmen im Wärmebereich als Schlüssel herausgestellt, um die 2010-Ziele zu erreichen. ${ }^{70}$ Auch das Grünbuch 2006 schlug

62 Vgl. Europäische Kommission: Communication from the Commission to the Council and the European Parliament: Green Paper follow-up action Report on progress in renewable electricity, COM(2006) 849 final, S. 5-10.

63 Vgl. Europäische Kommission: Communication from the Commission to the Council and the European Parliament: Renewable Energy Road Map, Renewable energies in the 21st century: building a more sustainable future, $\operatorname{COM}(2006) 848$ final.

64 Vgl. George Parker: Leaders leave tough bit until later: A deal has been achieved only by leaving the detail of 'burden-sharing' for others to sort out, in: Financial Times, 10.3.2007.

65 Vgl. Danyel Reiche/Mischa Bechberger: Diffusion von Einspeisevergütungen in der EU-25 als instrumenteller Beitrag zur Verbreitung erneuerbarer Energien, in: Mischa Bechberger/Danyel Reiche (Hrsg.): Ökologische Transformation der Energiewirtschaft, Erfolgsbedingungen und Restriktionen, Berlin 2006, S. 199-217.

$66 \mathrm{Vgl}$. Euractiv.com: Umweltkritik an Kommission wegen Rüge für Luxemburg, abrufbar unter: http://www.euractiv.com/de/energie/umweltkritik-kommission-wegen-ruge-luxemburg/article-158214 (letzter Zugriff: 31.5.07).

67 Vgl. Europäisches Parlament: Bericht über das Grünbuch „Energieeffizienz oder Weniger ist mehr“, A6-0160/ 2006.

68 Vgl. Stellungnahme von Paul Bulteel, Generalsekretär Eurelectric auf der Konferenz „Renewable Energy in the Internal Energy Market - The Way Forward", 18.04.2007, Brüssel.

69 Vgl. Europäische Kommission: Renewable Energy Road Map, 2006, S, 4.

70 Vgl. Europäische Kommission: Der Anteil erneuerbarer Energien in der EU, KOM(2204) 366 endgültig, 26.5.2004. 
eine neue Richtlinie für den Wärme- und Kühlbereich mit der Aussicht auf ein sektorales Ziel vor. Der Aktionsplan beinhaltet nun jedoch ein pauschales 20-Prozent-Ziel für erneuerbare Energien am Primärenergieverbrauch. Im Grünbuch 2006 hatte die Kommission außerdem einen Mindestanteil $\mathrm{CO}_{2}$-armer Energiequellen vorgeschlagen, der auch Kernenergie mit einbezogen hätte. ${ }^{71}$ Als sich im Vorlauf des EU-Ratstreffens abzeichnete, dass die deutsche Präsidentschaft - unterstützt durch Schweden, Dänemark, Österreich, Spanien sowie später Großbritannien - versuchen würde, einen verbindlichen Anteil erneuerbarer Energien am Energiemix der Mitgliedsländer zu vereinbaren, versuchten einige Länder - Frankreich, aber auch Tschechien und die Slowakei -, sich auf diese ursprüngliche Definition zu beziehen. ${ }^{72}$ Im Abschnitt 7 des Aktionsplans wird nun im Kontext der Forderung nach fairer Festlegung nationaler Gesamtziele auf die Abschnitte 10 und 11 verwiesen, die den Beitrag nachhaltigerer Nutzung fossiler Brennstoffe und der Kernenergie zur Versorgungssicherheit und Emissionsminderung thematisieren. Der französische Präsident Chirac wertete diesen Verweis auf die Kernenergie im Aktionsplan als Erfolg für Frankreich. ${ }^{73}$

Die Gipfelbeschlüsse zur erneuerbaren Energiepolitik mit globalen anstelle von sektoralen Zielen können als eine Art Renationalisierung der Energiepolitik in diesem Bereich interpretiert werden, da es im Rahmen nationaler Aktionspläne nunmehr den Mitgliedstaaten obliegt, sektorale Ziele festzulegen. Dadurch erhalten die Mitgliedstaaten Spielräume, bestimmte Technologien oder Ressourcen besonders zu fördern, wenngleich vorgesehen ist, dass die Kommission die nationalen Aktionspläne überprüft. Hinsichtlich der Umsetzung der Beschlüsse strebt die Kommission an, bis Ende 2007 einen Richtlinienentwurf vorzulegen. Wahrscheinlich werden Kommission und Mitgliedstaaten zu unterschiedlichen Bewertungen kommen, welcher Anteil erneuerbarer Energien jeweils vorgegeben wird. Dabei wird sich vor allem die Frage stellen, inwiefern $\mathrm{CO}_{2}$-arme Energieträger (vor allem Atomenergie) im bestehenden nationalen Energiemix in die Berechnungsmethode mit einbezogen werden.

Der Einfluss der Mitgliedstaaten auf die Umsetzung wird auch davon abhängen, auf welcher vertraglichen Basis innerhalb ihrer umweltpolitischen Kompetenz (Artikel 175 des EGVertrages) die Kommission vorgehen wird. Manche Mitgliedstaaten haben gefordert, dass der Gesetzgebungsprozess auf Absatz 2 beruhen muss, wonach der Rat einstimmig entscheidet und das Europäische Parlament nur beratende Funktion hat. Laut EG-Vertrag muss Absatz 2 angewendet werden, wenn ein Vorschlag bedeutenden Einfluss auf die Zusammensetzung der nationalen Energieversorgungsstruktur hat. Angesichts der proaktiven Rolle des Parlaments in diesem Politikfeld wäre ein Verfahren ohne volles Mitentscheidungsrecht des Parlamentes allerdings nur schwer durchsetzbar. Die Kommission bevorzugt daher ein Verfahren nach Absatz 1. Als Alternative wird innerhalb der Kommission auch darüber nachgedacht, den Vorschlag auf die Binnenmarkt-Kompetenzen der Kommission zu beziehen (Artikel 95 des EG-Vertrages). ${ }^{74}$ Dabei würde das Mitentscheidungsverfahren angewendet. Offen ist auch, ob das neue Regelwerk die bestehenden Richtlinien ersetzen und ob die angewandten Förderinstrumente harmonisiert werden sollen. Voraussetzung für eine Harmonisierung wäre jedoch ein wirklich integrierter Binnenmarkt. ${ }^{75}$

71 Vgl. Europäische Kommission: Eine europäische Strategie für nachhaltige, wettbewerbsfähige und sichere Energie, 2006, S. 11.

72 Vgl. Le Monde: Les Vingt-Sept se divisent sur le nucléaire et les énergies renouvelables, 7.03.2007.

73 Vgl. George Parker: Leaders leave tough bit until later, 2007.

74 Hans van Steen, Referatsleiter DG TREN, auf dem INFORSE/EREF European Sustainable Energy Policy Seminar, 20. März 2007, Brüssel.

75 Europäische Kommission: Report on progress in renewable electricity, 2006, S. 19. 


\section{Emissionshandel - auf zur ersten ,echten' Handelsperiode}

Im Jahre 2005 begann in der Europäischen Union die erste Handelsperiode des Europäischen Emissionshandelssystems für Energiewirtschaft und Industrie. Das System soll Anreize dafür setzen, dass Emissionsminderungen dort erfolgen, wo dies am günstigsten ist. Es wird als wesentliches Instrument zur Erreichung des europäischen Kyoto-Ziels angesehen. Von der (durch die EU-15) für die Periode 2008-2012 zugesagten Reduktion in Höhe von acht Prozent gegenüber 1990 waren bis 2005 lediglich 1,2 Prozent erreicht. ${ }^{76}$

Die im Dezember 2007 endende erste Handelsperiode kann im Wesentlichen als Lernphase bewertet werden. Deutliche Emissionsminderungen wurden nicht induziert, da die Zuteilung der Emissionsrechte in den meisten Ländern den Bedarf überstieg und daher aufgrund $\mathrm{zu}$ niedriger Zertifikatpreise kein Anreiz zu Emissionsreduktionen bestand. ${ }^{77}$ Zugleich entstanden bei vielen Energieversorgern deutliche Windfall-Profits, da die $\mathrm{CO}_{2}$ Zertifikate bei der Preisbildung an den Strombörsen - obgleich kostenlos zugeteilt - , eingepreist" wurden. ${ }^{78}$ Wesentliche Erfolge sieht die Kommission dennoch in der Etablierung eines aktiven Marktes sowie einer funktionierenden Infrastruktur. Gemäß Emissionshandelsrichtlinie $^{79}$ prüft die Kommission die von den Mitgliedsländern vorzulegenden Pläne zur Zuteilung der Zertifikate an die Emittenten. Für 21 Länder liegen inzwischen für die zweite Handelsperiode (die für das Kyoto-Protokoll relevante Verpflichtungsperiode 2008-2012) genehmigte und größtenteils stark gekürzte Pläne vor. ${ }^{80}$ Wie sich an den Preisen der heute gehandelten Zertifikate für die zweite Periode zeigt, gehen die Handelsteilnehmer hier von deutlich knapperen Rechten aus. Werden die von der Kommission genehmigten Pläne nicht durch Gerichtsentscheidungen revidiert, ${ }^{81}$ ist zu erwarten, dass der Emissionshandel ab 2008 beginnen kann, die intendierten Anreize zu setzen.

In den Schlussfolgerungen sowie im Aktionsplan wird die zentrale Rolle des Emissionshandels für die Verminderung der Treibhausgas-Emissionen betont. Es wird auf einen raschen Abschluss der derzeit von der Kommission durchgeführten Emissionshandels-Untersuchung gedrängt, um mehr Transparenz herzustellen und das System eventuell um die Sektoren Landnutzung und Forstwirtschaft sowie Land- und Schiffsverkehr zu erweitern. Für die Einbeziehung des Luftverkehrs ${ }^{82}$ wird bereits ein Richtlinienvorschlag erarbeitet. Die bisherigen Erfahrungen fließen auch in die Ausgestaltung der dritten Periode ab 2013 ein, wofür die Kommission in der zweiten Jahreshälfte 2007 einen Legislativvorschlag vorlegen will. Beinhalten könnte dieser - wie etwa vom Europäischen Parlament gefordert $^{83}$ eine stärkere Harmonisierung der Umsetzung und eine Begrenzung der kostenlosen Zuteilbarkeit der Rechte. Weiter wird angestrebt, das europäische System zum Nukleus eines welt-

76 Vgl. Deutsches Institut für Wirtschaftsforschung: Trotz Klimaschutzabkommen: Weltweit steigende $\mathrm{CO}_{2}-$ Emissionen, DIW-Wochenbericht Nr. 35/2006, S. 487.

77 Vgl. auch Europäische Kommission: EU-Emissionshandelssystem liefert erste überprüfte Emissionsdaten für Anlagen, Pressemitteilung, 15. Mai 2006.

78 Vgl. Tagesspiegel: Konzerne profitieren vom Klimaschutz, 29.06.2006.

79 Richtlinie 2003/87/EG in der Fassung der Richtlinie 2004/101/EG.

80 Vgl. Europäische Kommission: Emissionshandel: Kommission entscheidet über den nationalen Zuteilungsplan Italiens für 2008-2012, Pressemitteilung, 15. Mai 2007.

81 Polen und die Tschechische Republik haben angekündigt gegen die von der Kommission genehmigten gekürzten nationalen Allokationspläne vor dem Europäischen Gerichtshof Einspruch einzulegen.

82 Vgl. Europäische Kommission: Proposal for a Directive of the European Parliament and of the Council amending Directive 2003/87/EC so as to include aviation activities in the scheme for greenhouse gas emission allowance trading within the Community, $\operatorname{COM}(2006) 818,20.12 .2006$.

83 Europäisches Parlament: Beitrag zur Frühjahrstagung 2007. 
weiten Emissionshandels fortzuentwickeln beziehungsweise mit anderen Systemen zu verknüpfen. Interesse besteht hier etwa bei einigen Bundesstaaten der USA sowie in Australien. ${ }^{84}$

\section{Die strittige Rolle der Kernenergie}

Mit der Debatte um Klimawandel und Energieversorgungssicherheit werden nach einer langen Zeit der Zurückhaltung verstärkt wieder Argumente für eine Stärkung der Kernenergie vorgebracht. Der jüngste Bericht des Weltklimarates und die Internationale EnergieAgentur stufen Effizienz und erneuerbare Energien allerdings als bedeutendere Klimaschutzinstrumente ein. ${ }^{85}$ Die Kommission betont, die Kernenergie sei „eine der größten Quellen kohlendioxidfreier [...] Energie in Europa" ${ }^{\text {"86 }}$ und auch die europäische Stromindustrie befürwortet sie als Klimaschutzinstrument. ${ }^{87}$ Allerdings stimmt die Kommission der Internationalen Energie-Agentur zu, dass neue Kernkraftwerke in liberalisierten Märkten ohne staatliche Hilfen nicht zu erwarten sind. ${ }^{88}$

In den Niederlanden, Rumänien, Spanien, Großbritannien, Deutschland und der Tschechischen Republik liefert Kernenergie unter 30 Prozent (dem Durchschnitt der Europäischen Union) des Stroms. Über 50 Prozent beträgt der Kernenergieanteil dagegen in Belgien, der Slowakei, Litauen und dem Spitzenreiter Frankreich; ${ }^{89}$ zwölf Mitgliedsländer nutzen die Kernkraft nicht. Seit Anfang 2005 wurden in der Europäischen Union zehn Kernkraftwerke stillgelegt, die Stilllegung weiterer zehn wird bis 2010 erwartet. Die Anzahl der finanziell gesicherten und in Planung oder Bau befindlichen Neubauten liegt mit aktuell fünf deutlich niedriger. Da Kernkraftwerke lange Bau- und Planungszeiten haben - die Internationale Energie-Agentur geht von sieben bis 15 Jahren aus, vorausgesetzt, ein Land hat bereits alle notwendigen Nuklearinfrastruktur, ${ }^{90}$ müssten weitere Kernkraftwerke, die vor 2020 den kommerziellen Betrieb aufnehmen, heute bereits im fortgeschritteneren Planungsstadium sein. Entsprechend ist von einem Rückgang des Nuklearstrom-Anteils auszugehen.

Neben dem Verweis bezüglich der Erneuerbaren-Ziele der Mitgliedsländer taucht die Kernenergie in den Schlussfolgerungen des Europäischen Rates nur knapp auf. Das Kapitel V „Energietechnologien“ betont hier an erster Stelle die Entscheidungshoheit der Mitgliedstaaten über ihren Energiemix. Die Kommission übernimmt selbst keine direkten Initiativen, den Anteil der Kernenergie in Europa zu erhöhen; dies kritisierte das Europäische Parlament jüngst deutlich. ${ }^{91}$ Vielmehr stellen für sie eine Harmonisierung bei Sicherheit und Entsorgung sowie Forschung zu ,innovativen Reaktortechnologien“ eine Voraussetzung für die künftige Kernenergie-Entwicklung dar. ${ }^{92}$ Nachdem frühere legislative Vorstöße der Kommission nach

84 Vgl. Europäische Kommission: Mitteilung, $\operatorname{KOM}(2006)$ 676, 13.11.2006; Final Report of the 1st meeting of the ECCP working group on emissions trading on the review of the EU ETS on The Scope of the Directive on 8-9 March 2007, Brussels; Peter Zapfel: The EU emissions trading scheme as the driver for the global carbon market, Vortrag des EU-Emissionshandels-Koordinators der Kommission vom 22. Februar 2007.

85 Vgl. IPCC: Working Group III contribution to the IPCC Fourth Assessment Report. Summary for Policymakers, 2007, S. 25-33 und International Energy Agency: World Energy Outlook 2006, Paris 2006, S. 192.

86 Europäische Kommission: Eine Energiepolitik für Europa, 2007, S. 20.

87 Vgl. Eurelectric: Eurelectric welcomes new energy package, underlines key industry messages, 10.01.2007.

88 Vgl. Europäische Kommission: Mitteilung: Hinweisendes Nuklearprogramm, KOM(2006) 844, 10.01.2007 und International Energy Agency: World Energy Outlook 2006, S. 343.

89 Alle Daten: Bruttostromerzeugung, Quelle: Eurostat.

90 Vgl. International Energy Agency: World Energy Outlook 2006, S. 372.

91 Vgl. Europäisches Parlament: Bewertung von Euratom - 50 Jahre europäische Atomenergiepolitik, EntschlieBung P6_TA(2007)0181 vom 10. Mai 2007.

92 Vgl. Europäische Kommission: Hinweisendes Nuklearprogramm, 2007, S. 25. 
Artikel 119, Absatz 2 des Euratom-Vertrages hierzu gescheitert waren, ${ }^{93}$ soll nun die Einrichtung einer „High Level Group on Nuclear Safety and Waste Management“ etwas vorsichtiger auf ein gemeinsames Verständnis und später auf ergänzende europäische Regelungen hinarbeiten. Dieses Vorgehen wird vom Europäischen Rat unterstützt, ebenso wie die gemeinsamen Nuklearaktivitäten im Rahmen des 7. Forschungsrahmenprogramms. Zugleich wird vorgeschlagen, eine breite Debatte über die „Chancen und Risiken der Kernenergie“ zu führen.

\section{Die große Hoffnung auf die klimafreundliche Kohle}

Die Abscheidung von $\mathrm{CO}_{2}$ bei der Kohlestromerzeugung und dessen Speicherung in geologischen Formationen (Carbon Capture and Storage, CCS) erzeugen derzeit viel Aufmerksamkeit, da Kohle der weltweit noch am längsten verfügbare und zugleich preiswerteste, jedoch auch der klimaschädlichste fossile Brennstoff ist. ${ }^{94}$ Die Technologie befindet sich im Versuchsstadium, mit einer kommerziellen Verfügbarkeit wird nicht vor 2020 gerechnet. Recht sicher scheint jedoch bereits, dass die Technologie die Kraftwerkseffizienz senken und eher teuer werden wird. ${ }^{95}$ Sollte sich CCS als praktikabel erweisen, könnte dies künftige Klimaschutzverhandlungen mit China oder Indien - hier werden aktuell die meisten neuen Kohlekraftwerke errichtet ${ }^{96}$ - erleichtern. ${ }^{97}$

Die europäische Stromerzeugung basiert zu einem Drittel auf der Nutzung von Stein- und Braunkohle mit dem höchsten Kohleanteil am Primärenergieverbrauch ${ }^{98}$ in Polen (2004: 59 Prozent). ${ }^{99}$ Ein Ausstieg aus der Kohlenutzung würde für die europäische Energiewirtschaft einen großen Strukturwandel bedeuten. Entsprechend groß sind die Hoffnungen gerade bei den die Erzeugung dominierenden Stromversorgern auf diese Technologie. Sie dürfte aufgrund hoher Skalenerträge - wie die Kernenergie, und anders als die dezentrale Stromerzeugung in kleineren ${ }^{100}$ Einheiten - für kleinere Unternehmen kaum finanzierbar sein.

Das Vorantreiben der Kohlenstoffabscheidung hat für die Kommission eine hohe Priorität. Ziel ist, ab 2020 keine herkömmlichen Kohlekraftwerke mehr neu zu genehmigen. ${ }^{101}$ Auch das Parlament sieht hier ein wesentliches Klimaschutzinstrument. ${ }^{102}$ In ihrem Energiepaket kündigt die Kommission - dies begrüßt der Europäische Rat - die Förderung von bis zu zwölf Demonstrationsanlagen sowie eine eingehende Folgenabschätzung an. Überdies ist zu erwarten, dass sie - eventuell über verbindliche Rechtsvorschriften - bei vor 2020 neu errichteten Kohlekraftwerken auf eine hohe Effizienz und die Möglichkeit der Nachrüstung mit CCS-Technik drängen wird. ${ }^{103}$ Entsprechend der Aufforderung des Europäischen Rates

93 Vgl. Europäische Kommission: Geänderter Vorschlag vom 08.09.2004 für eine Richtlinie (Euratom) des Rates zur Festlegung grundlegender Verpflichtungen und allgemeiner Grundsätze im Bereich der Sicherheit kerntechnischer Anlagen. Geänderter Vorschlag für eine Richtlinie (Euratom) des Rates über die Entsorgung abgebrannter Brennelemente und radioaktiver Abfälle, KOM(2004) 526 endgültig.

94 Vgl. International Energy Agency: World Energy Outlook 2006, S. 125-136.

95 Vgl. Wuppertal Institut für Klima, Umwelt und Energie: Strukturell-ökonomisch-ökologischer Vergleich regenerativer Energietechnologien (RE) mit Carbon Capture and Storage (CCS), Wuppertal 2007.

96 Vgl. International Energy Agency: World Energy Outlook 2006, S. 125.

97 Vgl. Memo der Europäischen Kommission vom 10.01.2007: Kurs auf eine Zukunft mit $\mathrm{CO}_{2}$-armen fossilen Brennstoffen.

98 Der Primärenergieverbrauch beinhaltet neben der Stromerzeugung auch die von Wärme und Kraftstoffen.

99 Alle Daten: Eurostat und Europäische Kommission: Fact Sheets by Country, 2005.

100 Diese Einschränkung bezieht sich z.B. auf große Offshore-Windparks oder auch Solarkraftwerke in sonnenreichen Regionen Südeuropas.

101 Vgl. Handelsblatt: Ab unter die Erde, 03.05.2007

102 Vgl. Europäisches Parlament: Beitrag zur Frühjahrstagung 2007.

103 Vgl. Europäische Kommission: Mitteilung Nachhaltige Stromerzeugung aus fossilen Brennstoffen - Ziel: Weitgehend emissionsfreie Kohlenutzung nach 2020, $\operatorname{KOM(2006)~843,~10.01.2007.~}$ 
wird sie außerdem an einer Schaffung der technischen, wirtschaftlichen und ordnungspolitischen Rahmenbedingungen für den Einsatz von CCS, auch innerhalb des Emissionshandels, arbeiten.

\section{Zusammenfassung und Ausblick}

Insgesamt kann der auf dem Frühjahrstreffen der Staats- und Regierungschefs der Europäischen Union verabschiedete energiepolitische Aktionsplan gegenwärtig (noch) nicht als bedeutender Schritt auf dem Weg zu einer gemeinschaftlichen europäischen Energiepolitik bewertet werden. Zwar ist insbesondere die Verabschiedung der Ziele im Bereich der erneuerbaren Energien und des Klimaschutzes angesichts der Ausgangslage vor dem Gipfel durchaus als Verhandlungserfolg zu werten. Konfliktpotenziale und damit Skepsis hinsichtlich der Herausbildung einer gemeinschaftlichen Energiepolitik bestehen jedoch in zweierlei Hinsicht: Einerseits verspricht die Konkretisierung und Umsetzung der beschlossenen Ziele und Maßnahmen zumindest schwierig zu werden. Erst im Rückblick wird sich erweisen, ob die Gipfelbeschlüsse damit zu schwach oder aber ein Zwischenschritt auf dem Weg zu substanzielleren Fortschritten waren, der angesichts der erheblichen Differenzen nicht übersprungen werden konnte. Andererseits wird jedoch auch die ,Umsetzung' der auf dem Gipfel ausgeklammerten Kernfragen europäischer Energiepolitik - um nationale EnergieChampions, nationale Erdgassicherung, nationale Energiemixe sowie Zielkonflikte zwischen Versorgungssicherheit, Wettbewerb und Klimaschutz - weiterhin für Zündstoff und vor allem Inkonsistenzen sorgen.

Im Hinblick auf die Vollendung des europäischen Energiebinnenmarktes ist die anvisierte Abspaltung der Netzsparte von integrierten Versorgungsunternehmen - von vielen als kritisches Element auf dem Weg zu einem Energiebinnenmarkt erachtet - aufgrund nationaler Widerstände unwahrscheinlich. In diesem Zusammenhang wurde außerdem der Frage aus dem Weg gegangen, wie protektionistische nationale Tendenzen zum ,Schutz' nationaler Energieriesen mit einem wettbewerblichen Rahmen vereinbar sind. Während die Kommission weiter gegen solche Tendenzen ankämpft, zeigen sich die Mitgliedstaaten davon weitgehend unbeeindruckt. Als Hauptargument wird die Notwendigkeit großer ,Player ' vorgebracht, die für eine starke Verhandlungsposition auf den internationalen Energiemärkten unabdingbar seien - dass damit der Wettbewerb zurückstehen müsse, wird nicht offen diskutiert.

Solche nationalen Reflexe lassen eine europäische Energieaußenpolitik - ein Schlüsselelement des Aktionsplans - eher als Wunschdenken erscheinen. Die Beschlüsse zur Diversifizierung der Importregionen und -wege sind weitgehend eine Fortsetzung oder Verstärkung bestehender Initiativen. In unterschiedlichen strategischen Bewertungen der Mitgliedstaaten spiegeln sich unter anderem Unterschiede der nationalen Energieträgerstruktur wider. Zwar ist mit den zur Neige gehenden Öl- und Gasfeldern in der Nordsee eine Konvergenz der nationalen Interessen im Hinblick auf eine als Bedrohung wahrgenommene Importabhängigkeit festzustellen. Inwiefern sich dies aber in einem konvergenten Verhalten nach außen niederschlagen wird, bleibt abzuwarten. Nicht nur langfristige bilaterale Energielieferverträge stehen einem solchen ,Käuferkartell' (wie auch einem Gas-Kartell) im Wege, sondern auch das gezielt bilaterale Vorgehen der Energielieferländer. Die die energiepolitische Diskussion dominierende Sorge, dass die selbst während des Kalten Krieges stabilen Energielieferbeziehungen zwischen Westeuropa und Russland plötzlich verloren gehen könnten, sind einer sachlichen Betrachtung der Möglichkeiten und Grenzen einer europäischen Energieaußen- 
politik sicher nicht zuträglich, wie die ,Energiekrise‘ zwischen Russland und der Ukraine deutlich zeigte. ${ }^{104}$

In den Beschlüssen zur Energieeffizienzpolitik zeigt sich trotz der ambitionierten Ziele kein überzeugtes Vorgehen, was sich bereits an der mangelnden Implementation bestehenden europäischen Rechts manifestiert. Zwar erscheint es folgerichtig, zunächst auf dessen Umsetzung zu drängen, bevor wesentliche neue Rechtsakte in Angriff genommen werden. Jedoch besteht in diesem Bereich auch ein klarer Mangel an Evaluierung der Zielerreichung bestehender Maßnahmen.

Das als großer Erfolg der deutschen Ratspräsidentschaft gewertete verbindliche Ziel, bis 2020 einen 20-prozentigen Anteil erneuerbarer Energien am Primärenergieverbrauch zu erreichen, wird sich in einem komplizierten Aushandlungsprozess über , gerechte" nationale und sektorale Zielvorgaben bewähren müssen. Eine kritische Rolle werden ,kohlenstoffarme' Energieträger, allen voran die Kernenergie, spielen, deren Beitrag zum Klimaschutz Länder wie Frankreich bei der Berechnung der nationalen Ziele für erneuerbare Energien einbeziehen möchten. Zur Kernenergie selbst wurden auf dem Gipfel keine konkreten Beschlüsse gefasst, sondern die mögliche Einrichtung einer neuen Arbeitsgruppe vereinbart.

Neben den Beschlüssen zu erneuerbaren Energien wurde vor allem die Absichtserklärung, die Treibhausgasemissionen bis 2020 um 20 Prozent zu senken - mit der Aussicht auf 30 Prozent, sollten andere Industriestaaten folgen - als großer Erfolg gewertet, weil sie, etwa mit Blick auf das Treffen der G8 im Juni 2007, die Vorreiterrolle Europas im Klimaschutz unterstreichen sollte. Das geschlossene Auftreten der Europäer auf dem G8-Gipfel erwies sich als wichtiger Faktor, um die Post-Kyoto Verhandlungen im Rahmen der Vereinten Nationen voranzutreiben. Der beschlossene Aktionsplan begnügt sich jedoch damit, den Emissionshandel als zentral zu bezeichnen. Dabei zeigt sich gerade aktuell, dass ein wirkungsvoller Emissionshandel, der ambitionierte Emissionsreduktionen ermöglichen würde, von der Akzeptanz der von der Kommission geprüften nationalen Allokationspläne abhängt, um einen ausreichend hohen Zertifikatepreis zu gewährleisten. Nur wenn die zweite Handelsperiode ,funktioniert', werden sich die Ziele, einen globalen Kohlenstoffmarkt zu etablieren sowie eine weitere Vorreiterrolle Europas im Klimaschutz, erreichen lassen.

Im Hinblick auf das relativ starke Umsetzungsdefizit in der europäischen Energiepolitik stellt sich die Frage, ob nicht neue Politikansätze in diesem Politikbereich notwendig wären. Ähnlich wie in der europäischen Umweltpolitik ${ }^{105}$ könnte auch in Bezug auf eine europäische Energiepolitik versucht werden, diesem Umsetzungsdefizit mit neuen Politikinstrumenten oder ,policy modes“106 zu begegnen. Die jüngsten Beschlüsse deuten möglicherweise auf eine solche Veränderung hin: Die Koordinierung nationaler Behörden anstelle der Einrichtung einer europäischen Regulierungsbehörde, der europäische Strategieplan für Energietechnologien sowie die Einrichtung einer High Level Group im Bereich Kernenergie deuten auf eine Politik der Koordination hin. Ob dies ein effektiver Weg ist, die Umsetzung der europäischen Energiepolitik zu verbessern, bleibt abzuwarten.

Wie bereits in der Vergangenheit zeigte sich im Aushandlungsprozess um das Energiepaket und den Aktionsplan, dass sich die Prioritäten im Hinblick auf die Ziele einer gemein-

104 Vgl. Jonathan Stern: The new Security Environment for European Gas: Worsening Geopolitics and increasing Global Competition for LNG, Oxford Institute for Energy Studies 2006, S. 3-12, abrufbar unter: http:// www.oxfordenergy.org/pdfs/NG15.pdf (letzter Zugriff: 21.6.07).

105 Vgl. Andrea Lenschow: Environmental Policy - Contending Dynamics of Policy Change, in: Helen Wallace/ William Wallace/Mark A. Pollack (Hrsg.): Policy-Making in the European Union, Oxford 2005, S. 319-324.

106 Vgl. Helen Wallace: An Institutional Anatomy and Five Policy Modes, in: Helen Wallace/William Wallace/ Mark A. Pollack (Hrsg.): Policy-Making in the European Union, Oxford 2005, S. 77-89. 
schaftlichen Energiepolitik verschoben haben. Zugleich traten nationale Unterschiede wie auch Differenzen zwischen den EU-Institutionen zu Tage. Die Beschlüsse im Aktionsplan lassen kaum erkennen, dass die seit Langem notwendige klare Prioritätensetzung europäischer Energiepolitik angegangen wird. Während die Binnenmarktagenda immer noch dominant erscheint, drängen die Problemfelder Energiesicherheit und Klimaschutz zunehmend in den Vordergrund. Damit ergibt sich eine substanzielle Änderung im Handlungsfeld europäischer Energiepolitik von einem internen (mehr Wettbewerb) zu einem externen und eher transnationalen Fokus (Versorgungssicherheit und Klimaschutz) und folglich in den außenpolitischen Bereich. Kohärenz ist daher nicht nur zwischen den verschiedenen Zielen nach innen, sondern auch zwischen der internen und externen Dimension europäischer Energiepolitik notwendig.

Abschließend stellt sich im Hinblick auf die Umsetzung des Aktionsplans die Frage, inwiefern der Energiegipfel 2007 von einer, einmaligen ' Konstellation bestimmt war: Versorgungsstörungen mit Russland, Berichte zum Klimawandel, Sektoruntersuchung, Ratsvorsitz eines in Klimaschutzfragen eher progressiven Landes. Während dies ausreichte, ein Politikfenster zur Formulierung von zum Teil ehrgeizigen Zielen aufzustoßen, wird es sich in den kommenden Monaten zeigen, ob die Umsetzungsplanung Grund zur Hoffnung gibt, dass die angestrebten Ziele auch erreicht werden. Dabei wird der politische Wille der Mitgliedstaaten eine entscheidende Rolle spielen.

\title{
Neu im Programm
}

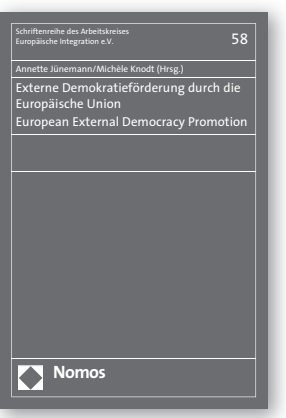

\author{
Externe Demokratieförderung durch \\ die Europäische Union \\ European External Democracy Promotion
}

Herausgegeben von Annette Jünemann und Michèle Knodt

2007, ca. 376 S., brosch., ca. 49,-€, ISBN 978-3-8329-2794-3

(Schriftenreihe des Arbeitskreises Europäische Integration e.V., Bd. 58)

Erscheint August 2007

Das Thema der externen Demokratieförderung durch die EU steht zur Zeit ganz oben auf der politischen Agenda, ist aber durch die Wissenschaft bisher kaum systematisch analysiert worden. In diese Forschungslücke stößt der vorliegende Band, der mögliche Erklärungen für die Strategie- und Instrumentenwahl der europäischen externen Demokratisierungspolitik in unterschiedlichen Staaten diskutiert. 